\title{
Spin-charge disparity of polarons in organic ferromagnets
}

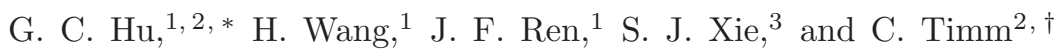 \\ ${ }^{1}$ College of Physics and Electronics, Shandong Normal University, Jinan 250014, China \\ ${ }^{2}$ Institute of Theoretical Physics, Technische Universität Dresden, 01062 Dresden, Germany \\ ${ }^{3}$ School of Physics, Shandong University, Jinan 250100, China
}

(Dated: May 28, 2013)

\begin{abstract}
Polaron formation in quasi-one-dimensional organic ferromagnets is studied based on an extended $\mathrm{Su}$-Schrieffer-Heeger model combined with a Kondo term. The charge distribution of the polaron is found to be highly asymmetric under spatial reflection, due to the spin radicals. On the contrary, the spin density is nearly symmetric; the spin asymmetry introduced by the extra electron inducing the polaron formation is nearly compensated by the spin polarization of the lower-energy states. We discuss these results on the basis of real-space mean-field calculations and symmetry arguments.
\end{abstract}

PACS numbers: 75.50.Xx, 71.38.-k, 73.61.Ph

Introduction.-Organic spintronics, i.e., the manipulation of electronic spin degrees of freedom in organic molecules, has attracted increasing interest in recent years [1 3]. One of the characteristic properties of organic materials is the strong electron-lattice interaction. This makes the ground state of conjugated organic materials dimerized. For example, trans-polyacetylene, trans$(\mathrm{CH})_{x}$, with uniform $\mathrm{C}-\mathrm{C}$ bond lengths would be a onedimensional metal with a half-filled band. However, the system can lower its energy by spontaneously forming a dimerized state with alternating short and long bonds [4, 5]. This Peierls instability opens a dimerization gap in the electronic spectrum, which induces the conjugated organic materials as organic semiconductors. The strong electron-lattice interaction also leads to another important effect: Electrons or holes doped into a polymer typically self-trap and form nonlinear excitations such as polarons and bipolarons [4]. A polaron consists of an electron (hole) with specific spin together with a localized distortion of the polymer chain. The whole polaron can move under an electric field as a quasiparticle, which is crucial for organic light-emitting diodes and organic spin-valve devices [6, 7].

Organic ferromagnets are particularly fascinating since they combine ferromagnetic and polaronic properties. Purely organic ferromagnets not containing transitionmetal ions can be realized by using spin radicals [8 12], which are usually heterocycles containing an unpaired electron [13]. For example, the organic ferromagnet polyBIPO can be obtained from polyacetylene by replacing every other $\mathrm{H}$ atom by a spin radical [8, 9]. There is an exchange coupling between the $\pi$ electrons in the main carbon chain and the residual spins of radicals. In the ground state, the radical spins order ferromagnetically [8, 9, 14, 15]. Recent progress in molecular electronics offers the prospect of designing spintronics devices based on organic ferromagnets, including spin filters [16, 17], spin diodes [18], and spin valves [19, 20]. This requires to understand the property of carriers in such materials, in which the spin coupling with radicals and the electron- lattice interaction will be important.

In this paper, we study the effects of adding an extra electron to a ferromagnetic polymer such as poly-BIPO. The extra electron forms a polaron, which is dramatically different from a polaron in a nonmagnetic chain. Specifically, its charge density is highly asymmetric, whereas its spin density, relative to the chain without polaron, is nearly symmetric. We show that this disparity is enhanced by the proximity of poly-BIPO to the transition to a periodic phase and discuss it in terms of the ease with which the charge and spin densities can adapt in order to lower the polaron energy.

Model and method.-We describe a quasi-onedimensional organic ferromagnet such as poly-BIPO. The molecule consists of a $\pi$-conjugated carbon chain and spin radicals attached to the odd sites. Each radical contains an uncompensated spin $\mathbf{S}_{i}$. We assume an antiferromagnetic coupling between the spin of the $\pi$ electrons and the radical spins $\mathbf{S}_{i}$. Such molecules can be well described by an extended Su-Schrieffer-Heeger (SSH) model [21] combined with a Kondo term. The Hamiltonian is written as [14, 15]

$$
\begin{aligned}
H= & -\sum_{i, \sigma}\left(t_{0}-\alpha y_{i}\right)\left(c_{i+1, \sigma}^{\dagger} c_{i, \sigma}+\text { H.c. }\right)+\frac{K}{2} \sum_{i} y_{i}^{2} \\
& +U \sum_{i} c_{i, \uparrow}^{\dagger} c_{i, \uparrow} c_{i, \downarrow}^{\dagger} c_{i, \downarrow}+J \sum_{i} \delta_{i \text { odd }} \mathbf{S}_{i} \cdot \mathbf{s}_{i} .
\end{aligned}
$$

The first term describes the hopping of $\pi$ electrons along the carbon chain. Here, $c_{i, \sigma}^{\dagger}\left(c_{i, \sigma}\right)$ denotes the creation (annihilation) operator of an electron at site $i$ with spin $\sigma$. $t_{0}$ is the hopping integral between two neighboring sites of a uniform chain and $\alpha$ denotes the electron-lattice coupling constant. $y_{i}$ is the lattice distortion $y_{i} \equiv u_{i+1}-u_{i}$, where $u_{i}$ is the displacement of the carbon atom at site $i$. The second term is the elastic energy due to the lattice distortion with elastic coefficient $K$. The third term is the on-site electron-electron interaction of strength $U$. The last term denotes the antiferromagnetic coupling between the $\pi$-electron spins $\mathbf{s}_{i}=\sum_{\sigma \sigma^{\prime}} c_{i, \sigma}^{\dagger}\left(\boldsymbol{\sigma}_{\sigma \sigma^{\prime}} / 2\right) c_{i, \sigma^{\prime}}$ 
and the radical spins $\mathbf{S}_{i}$, with strength $J>0$, and $\delta_{i \text { odd }}=1\left(\delta_{i \text { odd }}=0\right)$ for $i$ odd (even). Periodic boundary conditions are assumed.

The ground state of the molecule is calculated using a mean-field approximation for the spin-spin and electron-electron interactions. We obtain the eigenenergies $\varepsilon_{\mu, \sigma}$ and the eigenstates $\left|\psi_{\mu, \sigma}\right\rangle$ with (real) eigenfunctions $\psi_{\mu, \sigma, i}$ in Wannier space by solving the Schrödinger equation

$$
\begin{aligned}
& \varepsilon_{\mu, \sigma} \psi_{\mu, \sigma, i}=\sum_{j} H_{\sigma, i j}^{\mathrm{MF}} \psi_{\mu, \sigma, j} \\
& \quad \equiv-\left(t_{0}-\alpha y_{i}\right) \psi_{\mu, \sigma, i+1}-\left(t_{0}-\alpha y_{i-1}\right) \psi_{\mu, \sigma, i-1} \\
& \quad+U \bar{n}_{i,-\sigma} \psi_{\mu, \sigma, i}+J \delta_{i \text { odd }}\left\langle S_{i}^{z}\right\rangle \frac{\sigma}{2} \psi_{\mu, \sigma, i},
\end{aligned}
$$

where $\left\langle S_{i}^{z}\right\rangle$ is the average value of the radical spin, assumed to be in the $z$ direction, and $H_{\sigma, i j}^{\mathrm{MF}}$ is the matrix element of the mean-field Hamiltonian for the $\pi$ electrons with spin $\sigma$. The spin quantum number $\sigma$ assumes the numerical values $\uparrow \equiv 1$ and $\downarrow \equiv-1$. Throughout the paper, we enumerate the states such that $\varepsilon_{\mu^{\prime}, \sigma}>\varepsilon_{\mu, \sigma}$ for $\mu^{\prime}>\mu$. The average occupation number of $\pi$ electrons at site $i$ with spin $\sigma$ is $\bar{n}_{i, \sigma}=\sum_{\mu \text { occ. }}\left|\psi_{\mu, \sigma, i}\right|^{2}$. The sum is over all occupied states, i.e., all states with $\varepsilon_{\mu, \sigma}$ up to the Fermi energy. The lattice distortion $y_{i}=u_{i+1}-u_{i}$ in Eq. (2) is determined by minimizing the total energy

$$
E\left(\left\{y_{i}\right\}\right)=\sum_{\mu, \sigma \text { occ. }} \varepsilon_{\mu, \sigma}\left(\left\{y_{i}\right\}\right)+\frac{K}{2} \sum_{i} y_{i}^{2}
$$

with respect to the distortions $y_{i}$. The sum $\sum_{\mu, \sigma}$ occ. is over all occupied states. The contraint $\sum_{i} y_{i} \stackrel{=}{=} 0$ is implemented using a Lagrange multiplier. This leads to the equation

$$
\begin{aligned}
y_{i}= & -\frac{2 \alpha}{K} \sum_{\mu, \sigma \text { осc. }} \psi_{\mu, \sigma, i} \psi_{\mu, \sigma, i+1} \\
& +\frac{2 \alpha}{N K} \sum_{k} \sum_{\mu, \sigma \text { осс. }} \psi_{\mu, \sigma, k} \psi_{\mu, \sigma, k+1} .
\end{aligned}
$$

Equations (2) and (44) are solved self-consistently [14].

For the numerical calculations we use parameter values appropriate for poly-BIPO 14, 16, 22]: $t_{0}=2.5 \mathrm{eV}$, $\alpha=4.1 \mathrm{eV} / \AA, K=21.0 \mathrm{eV} / \AA^{2}$ and $\left\langle S_{i}^{z}\right\rangle=1 / 2$. We introduce dimensionless interaction strengths $j=J / t_{0}$ and $u=U / t_{0}$. The net charge density $\rho_{c, i}$ in units of the elementary charge $e>0$ and the spin density $\rho_{s, i}$ of $\pi$ electrons in the main chain, in units of $\hbar$, are defined as $\rho_{c, i} \equiv-\left(\bar{n}_{i, \uparrow}+\bar{n}_{i, \downarrow}-1\right)$ and $\rho_{s, i} \equiv\left(\bar{n}_{i, \uparrow}-\bar{n}_{i, \downarrow}\right) / 2$, respectively.

Neutral state.-Here, we consider a half-filled chain with $N=100$ sites. To focus on the effect of the spin radicals, we start with the case without electron-electron interactions, $u=0$. Without spin radicals $(j=0)$, the model reverts to a normal polymer. In the ground state the carbon atoms undergo a dimerization [4, 5] and a large energy gap (approximately $1.4 \mathrm{eV}$ ) opens up.

In the presence of spin radicals $(j>0)$, the radical spins order ferromagnetically due to their coupling to the $\pi$ electrons. As shown in previous numerical studies [14, 22], a spin density wave (SDW) is formed along the main chain, while the net charge density remains zero. This can be understood as follows: For fixed distortions $y_{i}$, the states $\left|\psi_{\mu, \sigma}\right\rangle$ are eigenstates of the mean-field Hamiltonian with eigenenergies $\varepsilon_{\mu, \sigma}$ and of the spin $z$ component with eigenvalues $\sigma / 2$. We construct new states $\left|\psi_{\mu^{\prime},-\sigma}\right\rangle$ by rotating the spin state by $180^{\circ}$ about the $x$ or $y$ axis and multiplying the wave function by $(-1)^{i}$, i.e., $\psi_{\mu^{\prime},-\sigma, i}=(-1)^{i} \psi_{\mu, \sigma, i}$. The mean-field Schrödinger equation (2) with $U=0$ shows that $\left|\psi_{\mu^{\prime},-\sigma}\right\rangle$ is an eigenstate with eigenenergy $\varepsilon_{\mu^{\prime},-\sigma}=-\varepsilon_{\mu, \sigma}$. According to our enumeration of states, we thus have $\mu^{\prime}=$ $N+1-\mu$. The charge density of the states $\left|\psi_{\mu^{\prime},-\sigma}\right\rangle$ and $\left|\psi_{\mu, \sigma}\right\rangle$ is clearly identical. The completeness relation ensures that the total charge density would be uniform, regardless of the distortions, if all states were occupied. In the neutral state, the chain is half filled. Thus, exactly one of the two states $\left|\psi_{\mu^{\prime},-\sigma}\right\rangle$ and $\left|\psi_{\mu, \sigma}\right\rangle$ is occupied. Since they have the same charge density, the total charge density of the half-filled chain is half that of the completely occupied chain and is thus uniform.

There is no analogous symmetry argument that protects the spin density. The spin density would vanish for a completely occupied chain, but since the states $\left|\psi_{\mu^{\prime},-\sigma}\right\rangle$ and $\left|\psi_{\mu, \sigma}\right\rangle$ have opposite spin density, the half-filled, neutral chain is not required to have vanishing spin density. As expected for antiferromagnetic coupling $j>0$, the spin density is negative at the odd sites, connected to the radical spins. However, the total spin in the neutral state is still zero, in spite of the radical spin preferring electronic spin down. To understand this, consider another state $\left|\psi_{\mu^{\prime \prime},-\sigma}\right\rangle$ that is obtained from $\left|\psi_{\mu, \sigma}\right\rangle$ by rotating the spin state by $180^{\circ}$ about the $x$ or $y$ axis and taking the mirror image of the wave function with respect to a bond center. This transformation maps short (long) bonds onto short (long) bonds but odd (even) sites onto even (odd) sites. We assume that the displacement $u_{i}$ is symmetric with respect to the mirror plane, which is the case for the periodic dimerized chain. Then it is straightforward to show using Eq. (2) with $U=0$ that $\left|\psi_{\mu^{\prime \prime},-\sigma}\right\rangle$ is also an eigenstate but with the eigenenergy $\varepsilon_{\mu^{\prime \prime},-\sigma}=\varepsilon_{\mu, \sigma}-\sigma J / 4$. The index is $\mu^{\prime \prime}=\mu$ since the mapping does not change the order of states. We see that for $J=0$ (no radicals), all eigenstates form degenerate pairs of opposite spin. For any even filling, the total spin vanishes. A nonzero exchange interaction $J>0$ lifts the degeneracy. However, the Fermi energy for the neutral state lies in the dimerization gap. As long as the energy splitting $J / 2$ is smaller than the dimerization gap, the states $\left|\psi_{\mu, \sigma}\right\rangle$ and $\left|\psi_{\mu^{\prime \prime},-\sigma}\right\rangle$ are still filled in pairs and the total spin remains zero. For the parameters used here, 

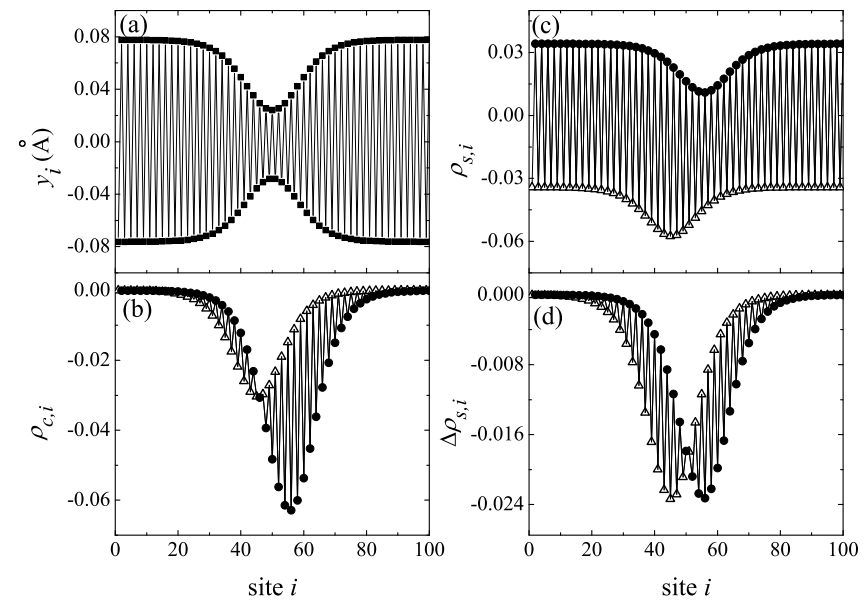

FIG. 1: Polaron state of the organic ferromagnet for exchange coupling $j=0.5$ : (a) Lattice distortion, (b) net charge density, (c) spin density, and (d) net spin density of the polaron calculated by subtracting the spin density of the neutral state from that of the charged one. Open triangles (filled circles) correspond to odd (even) sites. The same symbols are also used in the following figures.

this is satisfied.

Like in nonmagnetic polymers such as trans- $(\mathrm{CH})_{x}$, the short bonds can be either the even or the odd onesthe dimerization spontaneously breaks translation symmetry by doubling the unit cell, leading to two degenerate ground states. This is still the case for the magnetic polymer. However, now the dimerization in addition breaks reflection symmetry, the short bonds can be either to the left or to the right of a radical spin and these two ground states are not symmetric under spatial reflection.

Polaron state.-We now consider the state with one extra electron. From the previous discussion, one would expect it to occupy the lowest unoccupied molecular orbital (LUMO) with spin down. This is indeed found.

The exchange interaction is set to $j=0.5$. The lattice distortion, the net charge density, and the spin density are shown in Figs. 1(a)-1(c), respectively. It is clearly seen that the extra electron leads to the formation of a polaron. The distortion $y_{i}$ is reduced within the polaron - the dimerization is weaker there - and is symmetric with respect to the center of the polaron, within numerical accuracy. This is similar to the case of a normal polymer [23]. Additional insight can be gained by considering the net spin density $\Delta \rho_{s, i} \equiv \rho_{s, i}-\left.\rho_{s, i}\right|_{\text {neutral }}$ plotted in Fig. 1(d), obtained by subtracting the spin density of the neutral state from the one of the charged state. Compared to polarons in nonmagnetic polymers, two distinctive effects are observed: (i) The charge distribution is asymmetric with respect to the center of the polaron (the middle of the bond between sides 50 and 51 in the present case). Figure 1(b) shows that net charge density on both the odd and the even sites is peaked,

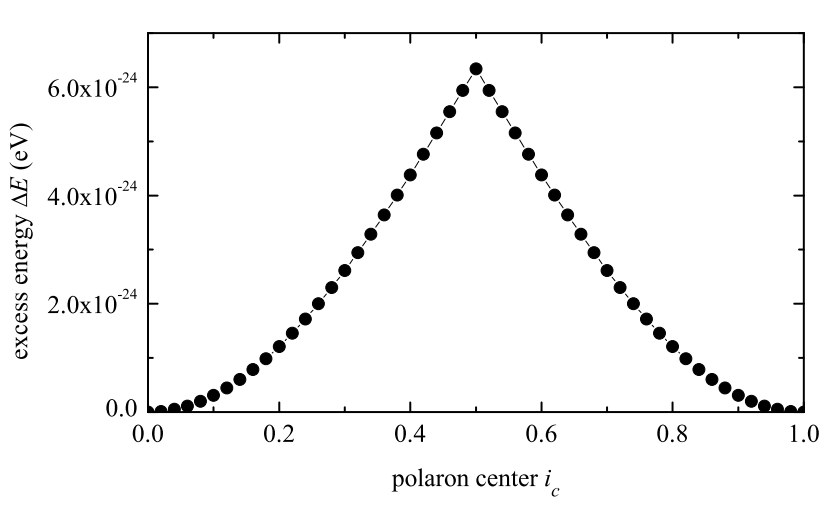

FIG. 2: Ground-state energy relative to its minimum of a polaron with constrained center $i_{c}$, as a function of $i_{c}$. Here, $i_{c}=0$ and 1 correspond to the bond-centered configuration, whereas $i_{c}=1 / 2$ corresponds to the site-centered configuration.

but with peak positions shifted to the left and right, respectively. Furthermore, the peak for the even sites is much larger than the one for the odd sites. (ii) The net (or excess) spin density does not coincide with the highly asymmetric charge density but instead is nearly symmetric; close examination of the data shows that there is a small asymmetry. Evidently the spin is distributed differently from the charge.

Moreover, in the ground state, the polaron is centered at a bond and the distortions are symmetric with respect to this center, see Fig. 1(a). This result is not trivial to obtain numerically because the energy differences involved are tiny. Figure 2 shows the energy as a function of the polaron center $i_{c}$. To obtain this quantity, we have minimized the total energy, Eq. (3), under the additional constraint of a fixed polaron center $i_{c}$. We write this constraint as

$$
C \sum_{k}(-1)^{k} y_{k}\left(\begin{array}{c}
\cos \frac{2 \pi k}{N} \\
\sin \frac{2 \pi k}{N}
\end{array}\right)=-\left(\begin{array}{c}
\cos \frac{2 \pi i_{c}}{N} \\
\sin \frac{2 \pi i_{c}}{N}
\end{array}\right),
$$

where $C$ is a normalization factor. This definition of $i_{c}$ implements the periodic boundary conditions by mapping the chain onto a ring. The factors $(-1)^{k}$ take care of the fact that the distortions are staggered, while the additional factor of -1 on the right-hand side is needed since the distortions are reduced in the region of the polaron so that the center of mass of $\left|y_{k}\right|$ is at the antipodal point of the polaron center. We implement Eq. (5) with additional Lagrange multipliers. Since the energy difference between polaron states with different $i_{c}$ are so small, we used Mathematica 24] with precision set to 32 digits to obtain Fig. 22 The energy differences are small because the polaron is relatively large, i.e., it comprises many sites, see Fig. 1. In the limit of diverging polaron size, a continuum model would become exact, which of course does not have any energy barrier for displacing 

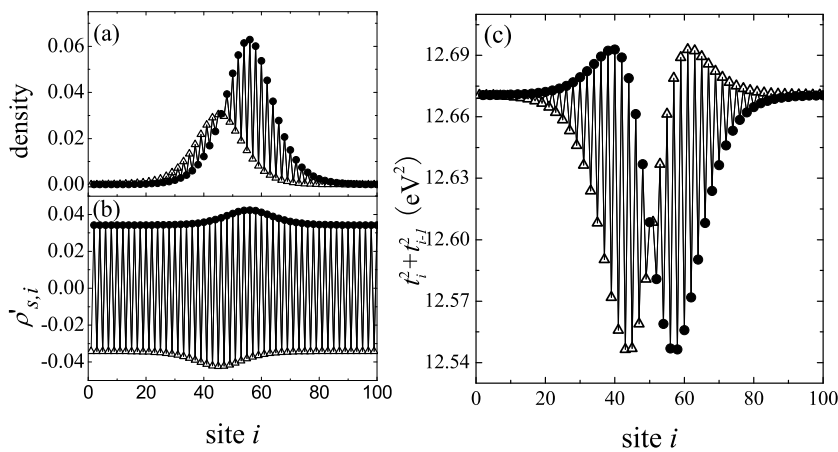

FIG. 3: (a) Probability density $\left|\psi_{\mu, \sigma, i}\right|^{2}$ of the orbital occupied by the additional spin-down electron. (b) Spin density $\rho_{s, i}^{\prime}$ contributed by the Fermi sea in the polaron state. (c) Sum of the square of hopping amplitudes $t_{i}=t_{0}-\alpha y_{i}$ between site $i$ and its neighbors.

the polaron.

To explain the anomalous spin and charge distribution, we first examine the wave function of the orbital $\left|\psi_{N / 2+1, \downarrow}\right\rangle$ occupied by the extra spin-down electron. Figure 2(a) shows the probability density of this orbital. The orbital has higher weight at the even sites compared to the odd sites, which is consistent with the charge distribution shown in Fig. प(b). The antiferromagnetic coupling actually favors spin-down electrons at the odd sites, which are the ones coupled to the radical spins. However, the orbital is antibonding so that its spatial distribution is opposite to the bonding states and has higher weight at the even sites. Indeed, it has the same probability density as its bonding spin-up partner state $\left|\psi_{\mu^{\prime}, \uparrow}\right\rangle$ constructed above.

In Fig. 3 (a), the maximum of the density on even (odd) sites is shifted to the right (left) with respect to the polaron center. This is correlated with the even bonds being long $\left(y_{i}>0\right)$ in the ground state found here, see Fig. 1(a). For the degenerate state with short even bonds we find the reversed pattern. The localization of the extra electron is due to the change in the hopping amplitudes $t_{i} \equiv t_{0}-\alpha y_{i}$. Figure 3(c) shows the quantity $t_{i}^{2}+t_{i-1}^{2}$ for each site, which is a measure of the hopping rate of an electron between site $i$ and its neighbors. In spite of a small increase for some sites, this quantity is obviously reduced in the polaron region. In particular, the largest reductions and thus the largest tendencies toward localization occur at sites displaced to the right (left) for even (odd) sites. This explains why the maximum density $\psi_{N / 2+1, \downarrow, i}^{2}$ on even (odd) sites is displaced to the right (left).

The spin and charge densities contributed by the orbital containing the extra electron are of course proportional to each other. Why, then, does the spin density of the polaron not follow the charge density? To analyze this, we have to consider also the other occupied states at lower energies. We here call these states the Fermi sea. First, we observe that the net charge density carried by the Fermi sea vanishes (not shown). This follows immediately from the argument given above for the uniform charge density in the neutral state - this argument works for arbitrary distortions $y_{i}$. Consequently, the charge density of the polaron is determined entirely by the single orbital considered above. On the other hand, the net spin density contributed by the Fermi sea, plotted in Fig. 3(b), clearly is affected by the polaron formation. The amplitude of the spin density wave is enhanced both for the odd (spin-down) and for the even (spin-up) sites but the maximum enhancements are shifted in opposite directions relative to the polaron center. The spin density $\rho_{s, i}^{\prime}$ of the Fermi sea is odd under reflection in a mirror plan through the polaron center, i.e., it has the symmetry property $\rho_{s, j}^{\prime}=-\rho_{s, i}^{\prime}$, where $j$ is obtained from $i$ by reflection. Evidently, the highly asymmetric net spin densities contributed by the orbital containing the extra electron and by the Fermi sea combine to form the nearly, but not quite, net symmetric spin density of the polaron.

In the following, we discuss these numerical findings. First, there is no symmetry that would require the spin density of the Fermi sea to vanish - it does not vanish even for the neutral state - or to be periodic for aperiodic distortions $y_{i}$. However, the spin density of the Fermi sea is odd under reflection with respect to the polaron center, see Fig. 3(b), and its total spin thus vanishes. This can be understood based on the second transformation discussed for the neutral case: The state $\left|\psi_{\mu^{\prime \prime},-\sigma}\right\rangle$ obtained from $\left|\psi_{\mu, \sigma}\right\rangle$ by spin rotation and spatial reflection with respect to a bond center is still an eigenstate in the polaron case, provided that this reflection leaves the distortions invariant. This requires the distortions to be symmetric with respect to the polaron center and in particular the polaron to be centered at a bond. This is the case, as we have shown above, see Fig. 2. The corresponding eigenenergy is $\varepsilon_{\mu^{\prime \prime},-\sigma}=\varepsilon_{\mu, \sigma}-\sigma J / 4$. As long as the exchange splitting $J / 2$ is smaller than the dimerization gap, which is the case for our parameters, in the Fermi sea either both or neither of the states $\left|\psi_{\mu^{\prime \prime},-\sigma}\right\rangle$ and $\left|\psi_{\mu, \sigma}\right\rangle$ are occupied. The spin densities of these two states are related by multiplication by -1 and spatial reflection. Their combined spin density is then invariant under this transformation. Consequently, the total spin density of the Fermi sea is odd under reflection. Thus the total spin of the Fermi sea vanishes.

Second, the question remains of why the asymmetric spin densities of the Fermi sea and of the extra electron combine to form a nearly symmetric net spin density, see Fig. 1(d). Figures 4(a) and 廿(b) show the net charge and spin densities of a polaron for the stronger exchange coupling $j=1.0$. The larger exchange coupling markedly increases the charge asymmetry but the spin asymmetry remains small. This begs the question whether the spin asymmetry might be smaller than the charge asymmetry 


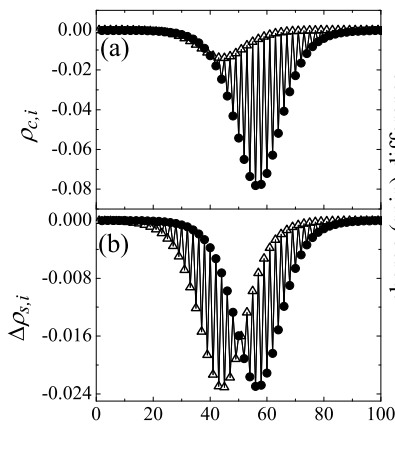

site $i$

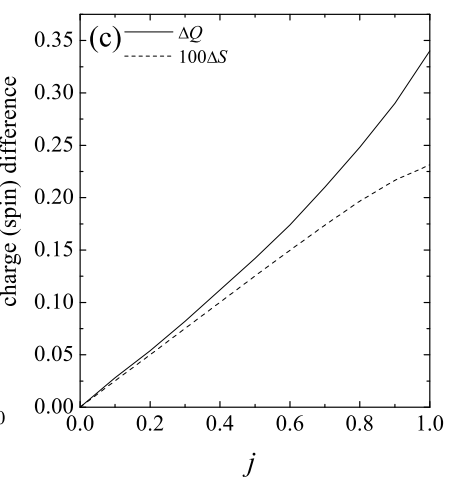

FIG. 4: (a) Net charge density and (b) net spin density of a polaron with a stronger exchange coupling $j=1.0$. (c) Charge asymmetry $\Delta Q$ (solid curve) and spin asymmetry $\Delta S$ (dashed curve) as functions of the exchange coupling $j$. The spin difference has been multiplied by 100 to make it more clearly visible.

because it is of higher order in the perturbation $j$ relative to the nonmagnetic chain. To answer this, it is useful to quantify the spin and charge asymmetries. Fixing the polaron center to the center of the bond between sites $N / 2$ and $N / 2+1$ without loss of generality, we define the spin and charge asymmetries as

$$
\begin{aligned}
& \Delta S \equiv\left|\sum_{i=N / 2+1}^{N} \Delta \rho_{s, i}-\sum_{i=1}^{N / 2} \Delta \rho_{s, i}\right| \\
& \Delta Q \equiv\left|\sum_{i=N / 2+1}^{N} \rho_{c, i}-\sum_{i=1}^{N / 2} \rho_{c, i}\right|
\end{aligned}
$$

respectively. Figure 4 (c) shows $\Delta S$ and $\Delta Q$ as functions of the exchange coupling $j$. Clearly, both asymmetries appear in first order in $j$. Thus an argument based only on perturbation theory in $j$ cannot explain the small spin asymmetry.

Hence, one might ask whether there is another small parameter. Such a small parameter indeed exists, namely the distance of the electron-lattice coupling $\alpha$ to its critical value $\alpha_{c}$, where polaron formation sets in. Figure 5 shows the spin and charge asymmetries as functions of $\alpha$, for otherwise the same parameters as above, in particular for a chain of length $N=100$. Note that the charge asymmetry increases strongly as the critical coupling strength $\alpha_{c}$ is approached from above, whereas the spin asymmetry decreases toward zero. For longer chains, the critical point $\alpha_{c}$ shifts to slightly lower values, the charge asymmetry increases even further (the downturn of $\Delta Q$ below $\alpha \approx 3.8 \mathrm{eV} / \AA$ in Fig. 5 is due to the polaron size becoming comparable with the chain length $N$ ), and the spin asymmetry still becomes small close to $\alpha_{c}$ (not shown). However, an additional phase with two solitons instead of one polaron intervenes between the polaron

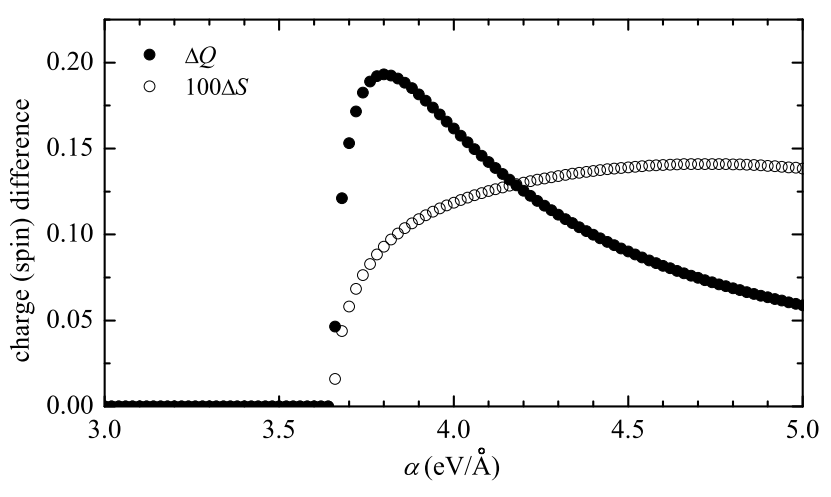

FIG. 5: Charge asymmetry $\Delta Q$ and spin asymmetry $\Delta S$ as functions of the electron-lattice coupling constant $\alpha$ for chain length $N=100$. The spin difference has been multiplied by 100 to make it more clearly visible.

and periodic phases. At the value of $\alpha=4.1 \mathrm{eV} / \AA$ assumed above, the dependence on the system size $N \geq 100$ is very weak.

The dependence on $\alpha$ shown in Fig. 5 suggests that the spin asymmetry is much smaller than the charge asymmetry due to the vicinity of $\alpha$ for poly-BIPO to the critical value. Why, then, is the spin asymmetry small for $\alpha \gtrsim \alpha_{c}$, whereas the charge asymmetry is large? The following is a plausible, though not rigorous, explanation. It is natural to expect the energy of a possible polaron configuration to increase with both the charge and the spin asymmetries, since if we impose a large (charge or spin) asymmetry as an additional constraint, the distortions $y_{i}$ have to adapt, which increases the elastic energy. This increase is not balanced by any term in the Hamiltonian (11) that would favor large asymmetries. However, the polaron cannot achieve a small charge asymmetry because the charge asymmetry is entirely due to the state occupied by the additional electron. Its width is controlled by the changes of the distortions $y_{i}$ in the polaron region, as shown above. For $\alpha \gtrsim \alpha_{c}$, the deviations of $y_{i}$ and thus of the hopping amplitudes $t_{i}=t_{0}-\alpha y_{i}$ from the periodically dimerized chain are small. On the other hand, the exchange coupling $J$ is constant and thus becomes strong relative to the aperiodic part of the $t_{i}$, which controls the localization. Hence, the extra electron, being in an antibonding state, will be nearly exclusively localized at even sites. But as discussed above, the maxima of the density for even and odd sites are displaced with respect to the polaron center in opposite directions, cf. Fig. 2(a). Thus the extra electron is mostly displaced in one direction for $\alpha \gtrsim \alpha_{c}$. In addition, the transition between the polaron state and the periodically dimerized state appears to be continuous or at most weakly first order so that the polaron size has to grow for $\alpha \rightarrow \alpha_{c}^{+}$. Hence, the extra electron is not only displaced mostly in one direction, it is also displaced by a large distance, leading to a large 


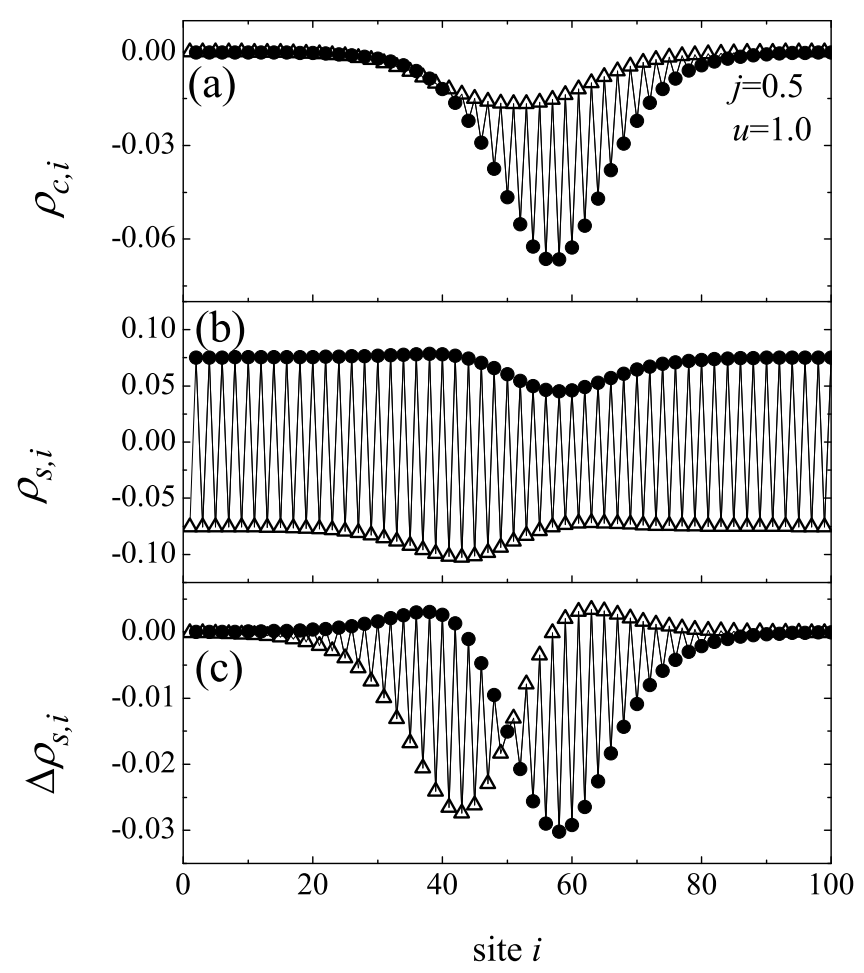

FIG. 6: Polaron state in the presence of electron-electron interaction: (a) Net charge density, (b) spin density and (c) net spin density of the polaron for $j=0.5$ and $u=1.0$.

charge asymmetry.

On the other hand, many states contribute to the spin asymmetry. The largest contributions come from occupied states close to the Fermi energy since states deep in the Fermi sea are little affected by the polaron formation. The number of contributing states increases with the polaron size - if the polaron size is comparable to the chain length $N$, all states are affected. Thus for $\alpha \rightarrow \alpha_{c}^{+}$, many states contribute. They are not subject to any simple constraint except that the total-spin $z$ component is $-1 / 2$. Based on our assumption that small asymmetries are energetically favorable, the polaron is therefore able to effectively minimize its energy by minimizing the spin asymmetry.

Electron-electron interaction.-We now examine the consequences of the electron-electron interaction. Here we set $u=1.0$, while the other parameters are same as used for Fig. 1] The results in Fig. [6 show that the picture of spin-charge disparity is robust even in the presence of electron-electron interaction. The effects of the electron-electron interaction are reflected in two aspects: First, the difference in charge density at even and odd sites is enhanced. Second, the amplitude of the oscillation in the spin density is increased both for the total and the net, localized contribution. This is because the repulsion of electrons with different spins on each site favors the formation of the SDW. It thus acts similarly to a stronger exchange coupling $J$. Other values for the electron-electron interaction have also been examined and the same picture is found provided that $u$ is not too large.

Finally, we should point out that the different behavior of spin and charge explored here is distinct from the well-known spin-charge separation in Luttinger liquids [25, 26], where the spin (spinon) and charge (holon) are different collective modes of the correlated electrons and generally move with different velocities. In our case, the observed spin-charge disparity is mediated by the polaronic lattice distortion, and of course both spin and charge are confined within the polaron. The motion of such a polaron under an external field can be expected to be nontrivial. We leave this issue to future work.

Summary.-We have investigated the spin and charge properties of polarons in quasi-one-dimensional organic ferromagnets with spin radicals attached to every second site, for example poly-BIPO. The results show spincharge disparity for the polarons: The charge density localized in the polaron region is highly asymmetric, whereas the spin density contributed by the polaron shows only very weak asymmetry. We attribute this disparity to the observation that the charge density of the polaron cannot efficiently adapt to minimize the polaron energy since it is carried by a single electronic state, whereas the spin density can adapt efficiently, being carried by many electrons. The effect is enhanced in the vicinity of the transition between the polaron state and the periodically dimerized phase, which is applicable for poly-BIPO.

Acknowledgments.- Support from the National Natural Science Foundation of China (Grant Nos. 10904084, 10904083, and 11174181), the Shandong Province Middle-Aged and Young Scientists Research Awards Foundation (Grant No. 2009BS01009), and the German Science Foundation, partially through Research Unit FOR 1154 "Towards Molecular Spintronics" are gratefully acknowledged. Helpful discussions with Dr. P. M. R. Brydon and Prof. Jianhua Wei are acknowledged.

* Electronic mail: hgc@sdnu.edu.cn

$\dagger$ Electronic mail: carsten.timm@tu-dresden.de

[1] W. J. M. Naber, S. Faez, and W. G. van der Wiel, J. Phys. D: Appl. Phys. 40, R205 (2007).

[2] V. A. Dediu, L. E. Hueso, I. Bergenti, and C. Taliani, Nature Mater. 707, 8 (2009).

[3] T. Sugawara and M. M. Matsushita, J. Mater. Chem. 19, 1738 (2009).

[4] A. J. Heeger, S. Kivelson, J. R. Schrieffer, and W. P. Su, Rev. Mod. Phys. 60, 781 (1988).

[5] R. E. Peierls, Quantum Theory of Solids (Clarendon Press, Oxford, 1955). 
[6] S. V. Rakhmanova and E. M. Conwell, Appl. Phys. Lett. 75, 1518 (1999).

[7] J. F. Ren, J. Y. Fu, D. S. Liu, L. M. Mei, and S. J. Xie, J. Appl. Phys. 98, 074503 (2005).

[8] Y. V. Korshak, T. V. Medvedeva, A. A. Ovchinnikov, and V. N. Spector, Nature (London) 326, 370 (1987).

[9] Y. Cao, P. Wang, Z. Hu, S. Z. Li, L. Y. Zhang, and J. G. Zhao, Synth. Met. 27, 625 (1988).

[10] H. Iwamura, T. Sugawara, K. Itoh, and T. Takui, Mol. Cryst. Liq. Cryst. 125, 379 (1985).

[11] Y. A. Katulevskii, M. A. Magrupov, and A. A. Muminov, Phys. Status Solidi A 127, 223 (1991).

[12] T. Sugano, S. J. Blundell, T. Lancaster, F. L. Pratt, and H. Mori, Phys. Rev. B 82, 180401 (2010).

[13] A. Alberola and M. Pilkington, Curr. Org. Synth. 6, 66 (2009).

[14] Z. Fang, Z. L. Liu, and K. L. Yao, Phys. Rev. B 49, 3916 (1994).

[15] Z. Fang, Z. L. Liu, K. L. Yao, and Z. G. Li, Phys. Rev. B 51, 1304 (1995).

[16] G. C. Hu, Y. Guo, J. H. Wei, and S. J. Xie, Phys. Rev. B 75, 165321 (2007).
[17] W. Z. Wang, Phys. Rev. B 73, 235325 (2006).

[18] G. C. Hu, K. L. He, S. J. Xie, and A. Saxena, J. Chem. Phys. 129, 234708 (2008).

[19] J. W. Yoo, R. S. Edelstein, D. M. Lincoln, N. P. Raju, C. Xia, K. I. Pokhodnya, J. S. Miller, and A. J. Epstein, Phys. Rev. Lett. 97, 247205 (2006).

[20] B. Li, M. Zhou, Y. Lu, C. Y. Kao, J. W. Yoo, V. N. Prigodin, and A. J. Epstein, Org. Electron. 13, 1261 (2012).

[21] W. P. Su, J. R. Schrieffer, and A. R. Heeger, Phys. Rev. Lett. 42, 1698 (1979).

[22] S. J. Xie, J. Q. Zhao, J. H. Wei, S. G. Wang, L. M. Mei, and S. H. Han, Europhys. Lett. 50, 635 (2000).

[23] W. P. Su and J. R. Schrieffer, Proc. Nat. Acad. Sci. U.S.A. 77, 5626 (1980).

[24] Wolfram Research, Inc., Mathematica, Version 8.0, Champaign, IL (2010).

[25] J. M. Luttinger, J. Math. Phys. 4, 1154 (1963).

[26] C. Kim, A. Y. Matsuura, Z. -X. Shen, N. Motoyama, H. Eisaki, S. Uchida, T. Tohyama, and S. Maekawa, Phys. Rev. Lett. 4, 4054 (1996). 